06

\title{
Формирование магнитных никелевых наночастиц и изменения матрицы фталоцианина никеля при допировании натрием
}

\author{
(C) Н.А. Колпачева, ${ }^{1,4}$ М.В. Авраменко, ${ }^{1}$ Л.А. Авакян, ${ }^{1}$ Я.В. Зубавичус, ${ }^{2}$ \\ А.А. Мирзаханян, ${ }^{3}$ А.С. Манукян, ${ }^{3}$ Э.Г. Шароян, ${ }^{3}$ Л.А. Бугаев ${ }^{1, \uparrow}$ \\ 1 Южный федеральный университет, \\ 344006 Ростов-на-Дону, Россия \\ ${ }^{2}$ Национальный исследовательский центр „Курчатовский институт“, \\ 123182 Москва, Россия \\ ${ }^{3}$ Институт физических исследований НАН Армении, \\ 0203 Гитаван-2, Аштарак, Армения \\ ${ }^{4}$ Донской государственный технический университет, \\ 344000 Ростов-на-Дону, Россия \\ ฯ e-mail: bugaev@sfedu.ru
}

(Поступило в Редакцию 27 декабря 2016 г.)

\begin{abstract}
Представлены результаты газофазного допирования фталоцианина никеля (NiPc) натрием с разной степенью допирования $(x)$, выполненного при $300^{\circ} \mathrm{C}$, а также результаты структурного анализа полученных образцов $\mathrm{Na}_{x=0.2} \mathrm{NiPc}, \mathrm{Na}_{x=1} \mathrm{NiPc}, \mathrm{Na}_{x=3} \mathrm{NiPc}$. Для изучения строения образцов и изменений их атомной структуры в зависимости от степени допирования использовались методы просвечивающей электронной микроскопии (TEM), спектроскопии комбинационного рассеяния света, рентгеновской дифракции (XRD) и рентгеновской абсорбционной спектроскопии (XAFS). Определены структурные параметры связей $\mathrm{Ni}-\mathrm{N}$, $\mathrm{Ni}-\mathrm{C}, \mathrm{Ni}-\mathrm{Ni}$, на основании которых установлено, что при малых дозах допирования натрием имеют место локальные структурные искажения части молекул фталоцианиновой матрицы в окрестности никеля, доля которых возрастает с увеличением степени допирования от $x=0.2$ до $x=1$. В результате допирования изменяется характер колебаний легких атомов, что указывает на появление дополнительной электронной плотности на пяти- и шестичленных кольцах. При высокой степени допирования натрием $(x=3)$ во фталоцианиновой матрице обнаружены никелевые наночастицы со средним размером около $20 \mathrm{~nm}$ и продукты распада молекул. Оцененная по данным XAFS доля атомов Ni в никелевых наночастицах от общего числа атомов $\mathrm{Ni}$ в $\mathrm{Na}_{x=3} \mathrm{NiPc}$ обеспечила наличие устойчивых на воздухе комнатно-температурных магнитных свойств образца, сохраняющихся длительное время.
\end{abstract}

DOI: 10.21883/JTF.2017.10.44998.2149

\section{Введение}

Наноразмерные магнитные наночастицы атомов переходных металлов ( $\mathrm{Me}=\mathrm{Fe}, \mathrm{Co}$, Ni и др.), стабилизированные в металлфталоцианиновой матрице $(\mathrm{MePc})$, представляют большой интерес в силу возможности их применения в оптико-электронных приборах, элементах органических полупроводников [1-4], в качестве нанокомпозитов для фотодинамической терапии [5], что обусловлено их способностью поглощать в ближней инфракрасной области. В последнее время тонкие пленки фталоцианинов, допированные электронами, вызывают большой интерес в молекулярной спинтронике, а также в сильно коррелированных электронных системах [6].

Магнитные свойства металлфталоцианинов, допированных щелочными металлами, изучались в работах [7-9], где было установлено, что при определенных условиях допирования образуются наночастицы металлов в матрице МеРс. Формирование наночастиц никеля в матрице NiPc при различных высоких степенях допирования атомами калия было изучено в работе [10], в которой для получения наночастиц использовался метод газофазного допирования $\mathrm{NiPc}$ калием при $300^{\circ} \mathrm{C}$.
Полученные материалы $\mathrm{K}_{x} \mathrm{NiPc}(x=1.8,2.5,3.2)$ демонстрировали сохраняющиеся во времени магнитные свойства, которые можно было связать с образовавшимися наночастицами никеля. В то же время образцы $\mathrm{NiPc}$, полученные нами при малых степенях допирования атомами щелочных металлов, также демонстрируют магнитные свойства, однако природа их происхождения остается открытой.

В настоящей работе представлены результаты газофазного допирования фталоцианина никеля NiPc натрием как с малой, так и с высокой степенью допирования, которое выполнялось при $300^{\circ} \mathrm{C}$, а также результаты структурного анализа синтезированных материалов. Методика получения образцов представлена в разд. 1 вместе с использованными методами диагностики их атомного строения: просвечивающей электронной микроскопии (ТЕМ), спектроскопии комбинационного рассеяния света (КРС), метода рентгеновской дифракции (XRD), рентгеновской абсорбционной спектроскопии в протяженной энергетической области (EXAFS). Использование такого подхода, сочетающего взаимодополняющие данные о синтезированном материале, полученные с помощью перечисленных методов исследования, поз- 
волило установить (разд. 2) наличие локальных структурных искажений в матрице NiPc при малых дозах допирования натрием, а также появление наночастиц никеля по мере увеличения степени допирования.

\section{1. Образцы и методы исследования}

Фталоцианин никеля синтезирован методом Линстеда из фталонитрила и соли $\mathrm{Ni}[11]$. Продукт реакции очищен промывкой в кислых и щелочных средах, после чего был дважды сублимирован в вакууме. Газофазное допирование [12] фталоцианина осуществлялось нагретыми до $300^{\circ} \mathrm{C}$ парами натрия. В результате ожидается формирование анионов молекул фталоцианина, согласно схеме

$$
\mathrm{NiPc}+x \mathrm{Na} \rightarrow\left(\mathrm{Na}^{+}\right)_{x}(\mathrm{NiPc})^{x-},
$$

где $x$ - степень допирования $(0<x<3)$. В случае более сильного допирования молекулы фталоцианина, как будет показано далее, разрушаются и образуются металлические наночастицы Ni. Допирование оказывается неоднородным, так что в образце могут сосуществовать области исходной фталоцианиновой матрицы, допированной матрицы и наночастиц никеля. После приготовления образцы были отожжены при температуре $280^{\circ} \mathrm{C}$ и промыты дистиллированной водой. Анализ элементного состава, выполненный энергодисперсионным спектрометром Inga-Energy 300, позволил установить средние значения степени допирования $x$ трех рассматриваемых образцов: $\mathrm{Na}_{x=0.2}(\mathrm{NiPc}), \mathrm{Na}_{x=1}(\mathrm{NiPc}), \mathrm{Na}_{x=3}(\mathrm{NiPc})$. Каждый из рассматриваемых образцов притягивается слабым постоянным магнитом.

ТЕМ-микрофотография образца $\mathrm{Na}_{x=3}(\mathrm{NiPc})$, представленная на рис. 1, получена с помощью электронного микроскопа FEI Tecnai F20.

Рентгеновские дифрактограммы и спектры рентгеновского поглощения за $K$-краем никеля (Ni $K$-XAFS) для чистого NiPc и образцов, допированных натрием, были измерены на источнике синхротронного излучения в НИЦ „Курчатовский институт“ (станция „Структурное материаловедение“) (Москва). Рентгеновские дифракционные профили измерены в режиме „на прохождение“ при длине волны излучения $0.1072 \mathrm{~nm} \mathrm{c} \mathrm{использо-}$ ванием детектора FujiFilm ImagePlate 2D. Измерения

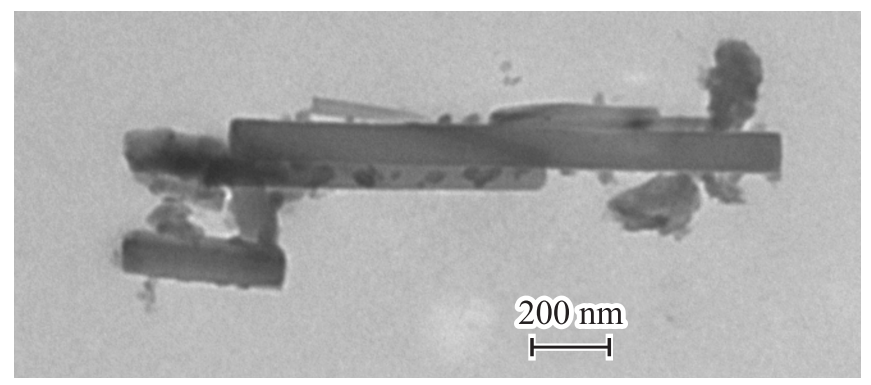

Рис. 1. ТЕМ-микрофотография образца $\mathrm{Na}_{x=3} \mathrm{NiPc}$.

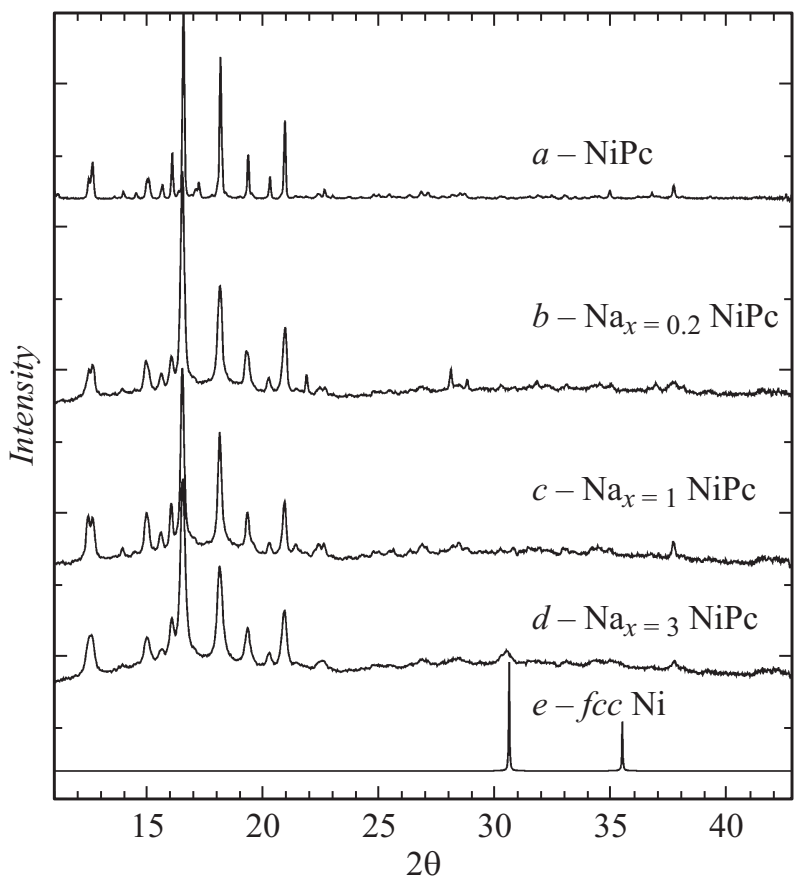

Pис. 2. XRD-профили образцов: $a-$ чистый NiPc, $b-$ $\mathrm{Na}_{x=0.2} \mathrm{NiPc}, c-\mathrm{Na}_{x=1} \mathrm{NiPc}, d-\mathrm{Na}_{x=3} \mathrm{NiPc}, e-$ фольга $\mathrm{Ni}$.

$\mathrm{Ni} K$-XAFS-спектров выполнены в режиме „на прохождение“ с использованием монохроматора $\mathrm{Si}(111)$ и двух ионизационных камер. Шаг сканирования был выбран $\delta E \sim 0.3 \mathrm{eV}$ для XANES ( $X$-ray absorption near edge structure) и $\delta k \sim 0.005 \mathrm{~nm}^{-1}$ для EXAFS (extended $X$-ray absorption fine structure) областей. Общее время получения одного спектра составило около $30 \mathrm{~min}$.

Спектры комбинационного рассеяния света (КРС) рассматриваемых образцов были получены в результате возбуждения колебаний аргоновым $(\lambda=514 \mathrm{~nm})$ или гелий-неоновым $(\lambda=633 \mathrm{~nm})$ лазером и регистрировались в геометрии обратного рассеяния спектрометром Renishaw, оснащенным CCD-детектором. В процессе измерений использовался 50-кратный объектив, фокусировавший лазерный луч на поверхности образцов в виде пятна диаметром около $2 \mu \mathrm{m}$.

\section{2. Результаты и обсуждение}

\section{1. ТЕМ-изображения, распределение по размерам и исследование дифракции рентгеновских лучей}

На рис. 1 представлена микрофотография образца $\mathrm{Na}_{x=3} \mathrm{NiPc}$, полученного с помощью метода TЕМ. Вытянутые стержни соответствуют кристаллиту (или агломератам кристаллитов) фталоцианина никеля. Более темные области с формой, близкой к сферической и средним размером $\sim 20 \mathrm{~nm}$, могут соответствовать наночастицам никеля. 


\section{2. Анализ XRD-профилей}

Рентгеновские дифракционные профили образцов $\mathrm{Na}_{x} \mathrm{NiPc}(x=0.2,1,3)$, представленные на рис. 2 вместе с профилями недопированного $\mathrm{NiPc}$ и никелевой фольги, демонстрируют появление хорошо выраженного рефлекса на угле $2 \theta \sim 30.5^{\circ}$ у образца $\mathrm{Na}_{x=3} \mathrm{NiPc}$, который отсутствует у недопированного $\mathrm{NiPc}$ и у образцов $\mathrm{Na}_{x=0.2} \mathrm{NiPc}, \mathrm{Na}_{x=1} \mathrm{NiPc}$. Сопоставление с пиками никелевой фольги позволяет заключить, что этот пик может быть отнесен к Ni ГЦК рефлексу отражения (111), что является признаком формирования наночастиц никеля. Пики при углах, меньших $2 \theta<25^{\circ}$, присутствуют как в чистом NiPc, так и у допированных образцов, а увеличение степени допирования приводит к уширению этих пиков. Поскольку чистый NiPc не демонстрирует металлических никелевых пиков, можно заключить, что наночастицы никеля действительно образуются в $\mathrm{NiPc}$ при степени допирования натрием $x \sim 3$.

В табл. 1 представлены результаты полнопрофильного анализа дифрактограмм образцов, который выполнялся на основе описанного выше качественного сопоставления с модельными объектами, т.е. с учетом двух базовых структур: фталоцианина никеля (P 12 / a 1 [13]) и наночастиц никеля $(F m 3 m[14])$. Согласно полученным результатам (табл. 1), в образцах $\mathrm{Na}_{x=0.2} \mathrm{NiPc}$ и $\mathrm{Na}_{x=1} \mathrm{NiPc}$ отсутствуют кристаллические частицы никеля, поскольку их концентрация оказывается менее погрешности ее определения. Однако в образце $\mathrm{Na}_{x=3} \mathrm{NiPc}$ наночастицы становятся заметными, и их доля составляет $3.0 \pm 0.3$ mass. $\%$.

\section{3. Результаты EXAFS}

Метод EXAFS спектроскопии был применен для подтверждения качественных выводов о структуре $\mathrm{Na}_{x} \mathrm{NiPc}$, сделанных на основе данных TEM- и XRD-измерений, a также для получения количественных характеристик структуры этих образцов. При построении моделей ближнего окружения атомов $\mathrm{Ni}$, используемых для фитинга фурье-образов $F(R)$ экспериментальных Ni $K$-EXAFS-спектров, было учтено, что в кристаллической структуре $\mathrm{NiPc}$, кратчайшее расстояние $\mathrm{Ni}-\mathrm{Ni}$ составляет $0.479 \mathrm{~nm}$ [13], что почти в 2 раза больше, чем длина $\mathrm{Ni}-\mathrm{Ni}$-связи в никелевой фольге $(0.249 \mathrm{~nm}[14])$. Благодаря такому различию оказывается возможным разделить вклады рассеяния фотоэлектрона на атомах никеля в наночастицах и в молекулярном кристалле. Для получения количественной информации о структуре ближнего окружения атомов $\mathrm{Ni}$ в образцах был выполнен анализ $\mathrm{Ni} K$-EXAFS-спектров, основанный на фурье-преобразовании (ФП) экспериментальных спектров и последующем фитинге полученных фурье-образов $F(R)$ в соответствии с методикой, предложенной в работе [15], позволяющей определять структурные параметры в условиях сильной корреляции между ними. С целью уменьшения влияния про- цессов многократного рассеяния (MS) на величины определяемых структурных параметров, нижняя граница $k$-интервала для ФП спектров была выбрана на значении $k_{\min }=0.45 \mathrm{~nm}^{-1}$ в соответствии с работой [16].

Для получения информации о структуре ближнего окружения атомов $\mathrm{Ni}$ в образце $\mathrm{Na}_{x=3} \mathrm{NiPc}$ использовалась модель, в рамках которой было учтено, что, согласно данным XRD-измерений, часть атомов Ni остается в составе молекул $\mathrm{NiPc}$, тогда как другая часть атомов Ni образует никелевые наночастицы. Обозначив вклад атомов $\mathrm{Ni}$ в составе молекул $\mathrm{NiPc} \chi_{\mathrm{NiPc}}(k)$, а вклад наночастиц (nanoparticles или NP) - как $\chi_{\mathrm{Ni}-\mathrm{NPs}}(k)$, можно записать в рамках используемой модели функцию $\chi(k)$ для EXAFS-спектра допированного образца в виде

$$
\chi(k)=C \chi_{\mathrm{Ni}-\mathrm{NPs}}(k)+(1-C) \chi_{\mathrm{NiPc}}(k),
$$

где $C$ - относительная концентрация поглощающих атомов $\mathrm{Ni}$, которые относятся к никелевым наночастицам. Расстояние между молекулярными слоями в структуре $\mathrm{NiPc}$ составляет около $0.35 \mathrm{~nm}$, что позволяет пренебречь вкладом межмолекулярного рассеяния и при анализе ФП $\mathrm{Ni} K$-EXAFS использовать модель фитинга для $\chi_{\mathrm{NiPc}}(k)$ в $(2)$, включающую только одну молекулу NiPc. Для уменьшения влияния процессов многократного рассеяния фотоэлектронов на определяемые параметры первой координационной сферы фитинг в $R$-пространстве был выполнен в интервале $1.0<R<0.35 \mathrm{~nm}^{-1}$ с помощью программного пакета IFEFFIT [17] для $F(R)$, полученной в результате ФП функции $k^{2} \chi(k)$ по интервалу $4.5 \leq k \leq 1.15 \mathrm{~nm}^{-1}$. Точность структурных параметров, полученных из Ni $K$-EXAFS с помощью такого подхода, протестирована в работе [10] на примере спектров

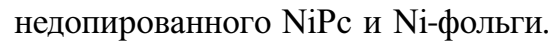

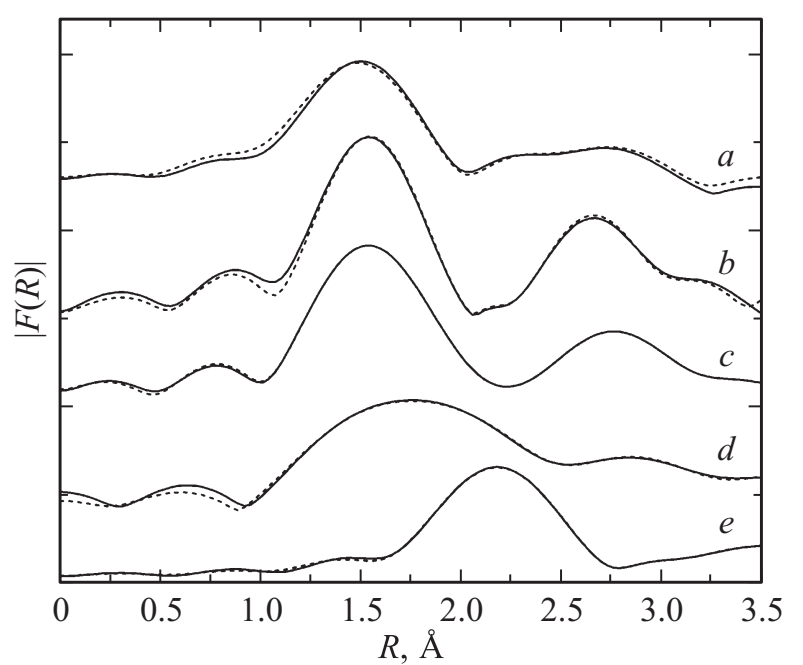

Рис. 3. Сравнение модулей фурье-образов $|F(R)|$ экспериментальных спектров Ni $K$-EXAFS (сплошные кривые) с результатами фитинга (пунктир). $a-$ чистый $\mathrm{NiPc}, b-\mathrm{Na}_{x=0.2} \mathrm{NiPc}$, $c-\mathrm{Na}_{x=1} \mathrm{NiPc}, d-\mathrm{Na}_{x=3} \mathrm{NiPc}, e-$ никелевая фольга. 
Таблица 1. Параметры кристаллических фаз в образцах $\mathrm{Na}_{x} \mathrm{NiPc}$ с различной степенью допирования $(x=0.2,1,3)$, полученные в результате анализа дифрактограмм: $C$ - весовая доля наночастиц никеля, $a, b, c, \beta-$ параметры решеток, $D-$ размер кристаллитов

\begin{tabular}{c|c|c|c|c|c|c|c|c|c}
\hline \multirow{2}{*}{ Образец } & \multirow{2}{*}{$R_{w p}, \%$} & \multicolumn{3}{|c|}{ Ni наночастицы } & \multicolumn{5}{c}{ NiPc } \\
\cline { 3 - 10 } & & $C$, w.\% & $a, \mathrm{~nm}$ & $D, \mathrm{~nm}$ & $a, \mathrm{~nm}$ & $b, \mathrm{~nm}$ & $c, \mathrm{~nm}$ & $\beta$ & $D, \mathrm{~nm}$ \\
\hline $\mathrm{Na}_{x=0.2} \mathrm{NiPc}$ & 5.8 & 0.4 & 0.374 & $\infty$ & 1.988 & 0.472 & 1.483 & $121.8^{\circ}$ & 83 \\
$\mathrm{Na}_{x=1} \mathrm{NiPc}$ & 5.3 & 0.5 & 0.374 & 30 & 1.992 & 0.471 & 1.484 & $121.8^{\circ}$ & 118 \\
$\mathrm{Na}_{x=3} \mathrm{NiPc}$ & 3.8 & 3.0 & 0.354 & 22 & 1.997 & 0.470 & 1.485 & $122.0^{\circ}$ & 66
\end{tabular}

Результаты обработки $\mathrm{Ni} K$-EXAFS в чистом NiPc и Ni-фольге позволили выполнить структурный анализ образца $\mathrm{Na}_{x=3} \mathrm{NiPc}$ по формуле (2) с использованием значений неструктурных параметров (факторы $S_{0}^{2}$ и энергетические параметры $\left.e_{0}[18]\right)$, полученных для этих эталонных образцов. Вклад никелевых наночастиц $\chi_{\mathrm{Ni}-\mathrm{NPs}}(k)$ в (2) включал вклады первых двух координационных сфер вокруг поглощающего атома $\mathrm{Ni}$ с различными параметрами: координационным числом $N_{\mathrm{Ni}-\mathrm{Ni}}$, радиусом координационной сферы $R_{\mathrm{Ni}-\mathrm{Ni}}$, параметром Дебая-Валера (ДВ) $\sigma_{\mathrm{Ni} \text {-Ni }}^{2}$, учитывающим тепловую и структурную разупорядоченность. Вклад $\chi_{\mathrm{NiPc}}(k)$ в (2) включал вклады первых трех сфер вокруг поглощающего атома $\mathrm{Ni}$ с варьируемыми для каждой сферы параметрами: $R$ и $\sigma^{2}$. Число $N$ атомов в каждой оболочке фиксировалось в соответствии с дифракционными данными для недопированного $\mathrm{NiPc}$, которые показывают сохранение большого числа молекул $\mathrm{NiPc}$ в допированном $\mathrm{Na}_{x=3} \mathrm{NiPc}$. Концентрация $C$, характеризующая долю атомов $\mathrm{Ni}$, входящих в состав Ni NPs, варьировалась как глобальный параметр. Сравнение $|F(R)|$ экспериментального Ni $K$-EXAFS в $\mathrm{Na}_{x=3} \mathrm{NiPc}$ с результатом фитинга, выполненного по описанной схеме, представлено на рис. 3, $d$, вместе с $|F(R)|$ для $\mathrm{NiPc}$ (рис. 3, $a$ ), $\mathrm{Ni-фольги} \mathrm{(рис.} 3, e)$ и результатами их фитинга, взятыми из работы [10]. Соответствующие структурные параметры ряда ближайших к поглощающему атому $\mathrm{Ni}$ координационных сфер в наночастицах и в молекулах $\mathrm{NiPc}$, полученные из EXAFS, представлены в табл. 2.

Данные табл. 2 показывают, что длины $\mathrm{Ni}-\mathrm{N}$-связей в $\mathrm{Na}_{x=3} \mathrm{NiPc}$ согласуются с недопированным $\mathrm{NiPc}$ в пределах ошибок измерений. Полученные значения параметров ДВ $\sigma^{2}$ для ближайших соседей $\mathrm{Ni}$ как в $\mathrm{Ni}$-наночастицах, так и в молекулах $\mathrm{NiPc}$ соответствуют комнатным температурам [19]. Такое соответствие фитинга свидетельствует о том, что используемая модель, основанная на уравнении (2), верно учитывает основные типы локального окружения поглощающих атомов $\mathrm{Ni}$ в $\mathrm{Na}_{x=3} \mathrm{NiPc}$. Представленные в табл. 2 параметры однозначно свидетельствуют о наличии наночастиц $\mathrm{Ni}$ в допированном образце $\mathrm{Na}_{x=3} \mathrm{NiPc}$ и показывают, что атомная структура оставшихся молекул NiPc существенно не изменяется при допировании натрием. Концентрация $C$ атомов $\mathrm{Ni}$, относящаяся к наночастицам $\mathrm{Ni}$, составляет $\sim 60 \%$.
Таблица 2. Параметры структуры ближнего окружения атомов Ni для различных фазовых состояний никеля в образце $\mathrm{Na}_{x=3} \mathrm{NiPc}$. Для сравнения в круглых скобках приведены значения соответствующих параметров в недопированном $\mathrm{NiPc}$ и в Ni-фольге

\begin{tabular}{|c|c|c|c|c|c|c|}
\hline Образец & $\begin{array}{c}\text { Структурное } \\
\text { состояние }\end{array}$ & $C, \%$ & Связи & $N$ & $R, \mathrm{~nm}$ & $\sigma^{2}, \mathrm{~nm}^{2}$ \\
\hline \multirow[t]{5}{*}{$\mathrm{Na}_{x=3} \mathrm{NiPc}$} & \multirow[t]{3}{*}{$\mathrm{NiPc}$} & \multirow[t]{3}{*}{40} & $\mathrm{Ni}-\mathrm{N}$ & 4.0 & $\begin{array}{c}0.191 \\
(0.191)\end{array}$ & $3 \cdot 10^{-5}$ \\
\hline & & & $\mathrm{Ni}-\mathrm{C}$ & 8.0 & $\begin{array}{c}0.305 \\
(0.295)\end{array}$ & $4 \cdot 10^{-5}$ \\
\hline & & & $\mathrm{Ni}-\mathrm{N}$ & 4.0 & $\begin{array}{c}0.318 \\
(0.335)\end{array}$ & $1 \cdot 10^{-5}$ \\
\hline & \multirow[t]{2}{*}{ NiNPs } & \multirow[t]{2}{*}{60} & $\mathrm{Ni}-\mathrm{Ni}$ & 5.4 & $\begin{array}{c}0.257 \\
(0.248)\end{array}$ & $2.5 \cdot 10^{-5}$ \\
\hline & & & $\mathrm{Ni}-\mathrm{Ni}$ & 2.7 & $\begin{array}{c}0.366 \\
(0.352)\end{array}$ & $1.8 \cdot 10^{-5}$ \\
\hline
\end{tabular}

Аналогичный фитинг для образцов $\mathrm{Na}_{x=0.2} \mathrm{NiPc}$ и $\mathrm{Na}_{x=1} \mathrm{NiPc}$ не выявил наличие $\mathrm{Ni}-\mathrm{Ni}$-связей, что указывает на отсутствие никелевых наночастиц при данных степенях допирования этих образцов. Такой вывод подтверждается результатами XRD-анализа и TEM-измерений. Между тем невысокое качество фитинга, основанного на выражении (2), в котором для воспроизведения вклада $\mathrm{NiPc}$ использовался только один тип $\mathrm{Ni}-\mathrm{N}$-связи, позволил предположить появление локальных искажений в первой координационной сфере $\mathrm{Ni}$, состоящей из атомов $N$, молекул NiPc. Поэтому фитинг $F(R)$ для Ni $K$-EXAFS в $\mathrm{Na}_{x=0.2} \mathrm{NiPc}_{\text {и }} \mathrm{Na}_{x=1} \mathrm{NiPc}$ выполнялся с использованием $\chi_{2}(k)$, определенной выражением

$$
\chi_{2}(k)=C \chi_{(\mathrm{Ni}-\mathrm{N}) 1}(k)+(1-C) \chi_{\mathrm{NiPc}}(k),
$$

в котором первое слагаемое $C \chi_{(\mathrm{Ni}-\mathrm{N}) 1}(k)$ соответствует искаженным структурам $\mathrm{Ni}-4 \mathrm{~N}$, концентрация или доля которых $-C$, а второе слагаемое $(1-C) \chi_{\mathrm{NiPc}}(k)-$ неискаженным молекулам NiPc.

Параметры структуры ближнего окружения Ni в образцах $\mathrm{Na}_{x=0.2} \mathrm{NiPc}$ и $\mathrm{Na}_{x=1} \mathrm{NiPc}$ с искаженной и неискаженной $\mathrm{Ni}-4 \mathrm{~N}$-структурами представлены в табл. 3. 
Таблица 3. Параметры структуры ближнего окружения $\mathrm{Ni}$ в образцах $\mathrm{Na}_{x=0.2} \mathrm{NiPc}$ и $\mathrm{Na}_{x=1} \mathrm{NiPc}$ с искаженной и неискаженной $\mathrm{Ni}-4 \mathrm{~N}$-структурами

\begin{tabular}{|c|c|c|c|c|c|c|}
\hline Образец & $\begin{array}{c}\text { Структурное } \\
\text { состояние }\end{array}$ & $C, \%$ & Связи & $N$ & $R, \mathrm{~nm}$ & $\sigma^{2}, \mathrm{~nm}^{2}$ \\
\hline \multirow[t]{4}{*}{$\mathrm{Na}_{x}=0.2 \mathrm{NiPc}$} & $\begin{array}{l}\mathrm{NiPc} \mathrm{c} \mathrm{искаженной} \\
\text { структурой } \mathrm{Ni}-4 \mathrm{~N}\end{array}$ & $\begin{array}{c}35.0 \\
\pm 5\end{array}$ & $(\mathrm{Ni}-\mathrm{Ni})_{1}$ & 4.0 & $\begin{array}{c}0.199 \\
(0.191)\end{array}$ & $10 \cdot 10^{-5}$ \\
\hline & \multirow[t]{3}{*}{ неискаженный NiPc } & \multirow[t]{3}{*}{$\begin{array}{c}65.0 \\
\pm 5\end{array}$} & $(\mathrm{Ni}-\mathrm{N})_{2}$ & 4.0 & $\begin{array}{c}0.189 \\
(0.191)\end{array}$ & $3 \cdot 10^{-5}$ \\
\hline & & & $\mathrm{Ni}-\mathrm{C}$ & 8.0 & $\begin{array}{c}0.292 \\
(0.295)\end{array}$ & $4 \cdot 10^{-5}$ \\
\hline & & & $\mathrm{Ni}-\mathrm{N}$ & 4.0 & $\begin{array}{c}0.331 \\
(0.335)\end{array}$ & $4 \cdot 10^{-5}$ \\
\hline \multirow[t]{4}{*}{$\mathrm{Na}_{x=1} \mathrm{NiPc}$} & $\begin{array}{l}\mathrm{NiPc} \text { с искаженной } \\
\text { структурой } \mathrm{Ni}-4 \mathrm{~N}\end{array}$ & 45.0 & $(\mathrm{Ni}-\mathrm{N})_{2}$ & 1.0 & $\begin{array}{c}0.203 \\
(0.191)\end{array}$ & $0.3 \cdot 10^{-5}$ \\
\hline & \multirow[t]{3}{*}{ Неискаженный NiPc } & \multirow[t]{3}{*}{$\begin{array}{c}55.0 \\
\pm 5\end{array}$} & $(\mathrm{Ni}-\mathrm{N})_{1}$ & 4.0 & $\begin{array}{c}0.189 \\
(0.191)\end{array}$ & $1 \cdot 10^{-5}$ \\
\hline & & & $\mathrm{Ni}-\mathrm{C}$ & 8.0 & $\begin{array}{c}0.293 \\
(0.295)\end{array}$ & $3 \cdot 10^{-5}$ \\
\hline & & & $\mathrm{Ni}-\mathrm{N}$ & 4.0 & $\begin{array}{c}0.332 \\
(0.335)\end{array}$ & $2 \cdot 10^{-5}$ \\
\hline
\end{tabular}

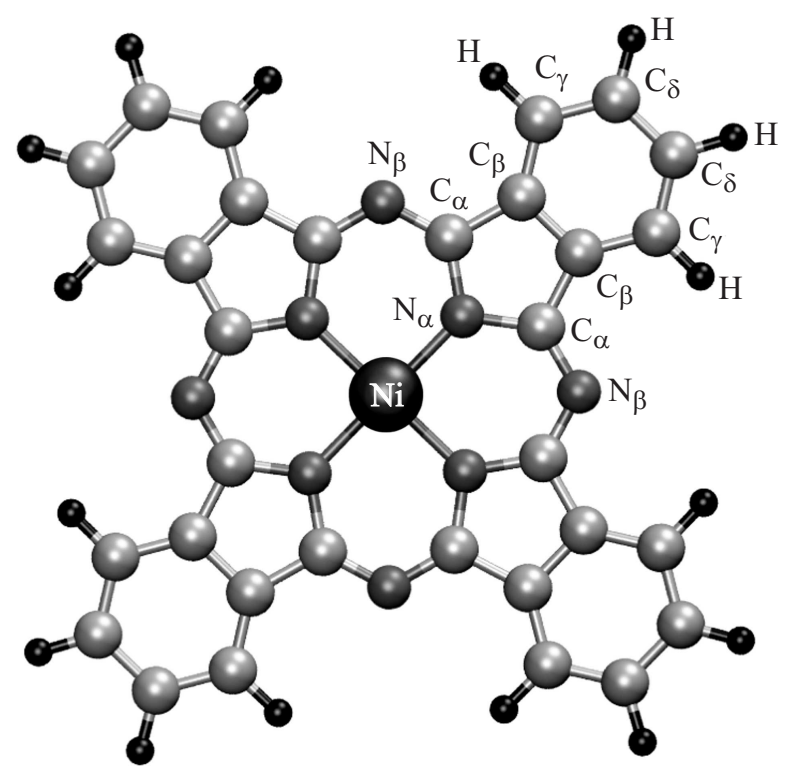

Рис. 4. Схематическое изображение структуры молекулы NiPc. Различные позиции атомов азота и углерода обозначены в соответствии с работой [20].

Отсутствие наночастиц $\mathrm{Ni}$ в образцах $\mathrm{Na}_{x=0.2} \mathrm{NiPc}$, $\mathrm{Na}_{x=1} \mathrm{NiPc}$ и их появление в образце $\mathrm{Na}_{x=3} \mathrm{NiPc}$ отчетливо наблюдается по динамике формы и положения первого главного пика на рис. 4, который сравнивает фурье-образы $|F(R)|$ осциллирующих частей $\chi(k)$ экспериментальных Ni $K$-EXAFS-спектров в чистом NiPc, в образцах $\mathrm{Na}_{x=0.2} \mathrm{NiPc}, \mathrm{Na}_{x=1} \mathrm{NiPc}, \mathrm{Na}_{x=3} \mathrm{NiPc}$ и никелевой фольге.

\section{4. Результаты спектроскопии КРС}

Структура молекулы NiPc схематически представлена на рис. 4. Согласно [20], симметрия данной молекулы описывается группой $D_{4 h}$, и результат фактор-группового анализа ее нормальных колебаний может быть представлен в виде: $14 A_{1 g}+13 A_{2 g}+14 B_{1 g}+$ $+14 B_{2 g}+13 E_{g}+6 A_{1 u}+8 A_{2 u}+7 B_{1 u}+28 E_{u}$. Моды, активные в спектрах КРС, имеют симметрию $A_{1 g}, B_{1 g}$, $B_{2 g}$ и $E_{g}$. При этом в работе [21] утверждается, что в спектрах КРС в интервале от 300 до $1650 \mathrm{~cm}^{-1}$ наблюдаются линии, соответствующие колебаниям макроциклического фталоцианинового кольца, а интенсивные линии, соответствующие колебаниям алифатических заместителей, отсутствуют. В свою очередь, колебания, в которых участвует центральный атом металла, могут наблюдаться в интервале частот около $200-300 \mathrm{~cm}^{-1}$.

На рис. 5, $a, b$ представлены спектры КРС, полученные при длинах возбуждающего излучения 514 и $633 \mathrm{~nm}$ соответственно. Между полученными спектрами КРC чистого NiPc и литературными данными [21,22] имеется ряд отличий, заключающихся как в разном числе линий, так и в их положении. Эти отличия могут быть обусловлены тем, что рассматриваемые образцы являются порошками, а в указанных работах изучались пленки.

Для всех образцов и длин волн области, соответствующие колебаниям атомов никеля, оказываются практически идентичными, что не позволяет сделать вывод об искажении локальной атомной структуры напрямую. При длине волны излучения $\lambda=514 \mathrm{~nm} \mathrm{(рис.} \mathrm{5,} a$ ) 


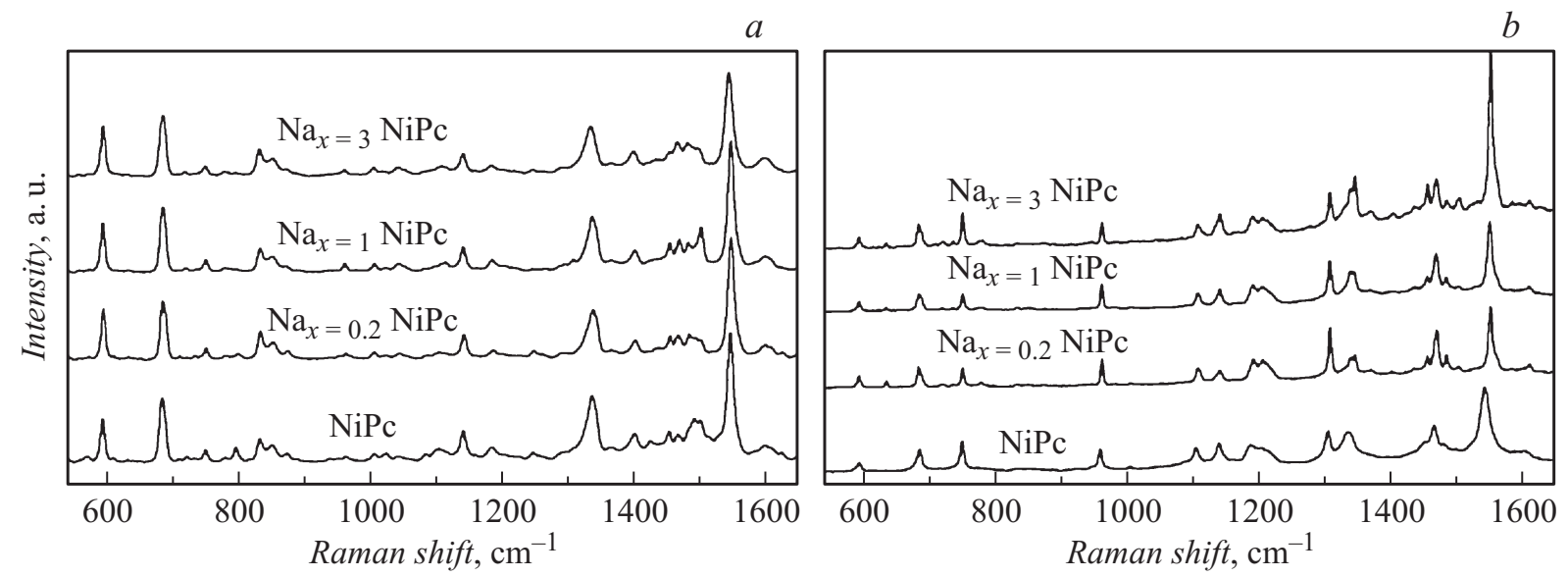

Рис. 5. Сравнение спектров КРС, полученных для образцов $\mathrm{NiPc}, \mathrm{Na}_{x=0.2} \mathrm{NiPc}, \mathrm{Na}_{x=1} \mathrm{NiPc}, \mathrm{Na}_{x=3} \mathrm{NiPc}$ при различных длинах волн возбуждающего излучения: $a-514, b-633 \mathrm{~nm}$. Спектры нормированы по интенсивности линии: $a-593, b-961 \mathrm{~cm}^{-1}$.

Таблица 4. Изменения в спектрах КРС, происходящие в результате допирования фталоцианина никеля натрием. Атомы, ответственные за колебания, обозначены в соответствии с рис. 4

\begin{tabular}{|c|c|c|}
\hline \multirow{7}{*}{$\begin{array}{c}\text { Частотные } \\
\text { сдвиги, } \mathrm{cm}^{-1}, \\
\text { (интерпретация) }\end{array}$} & $\mathrm{Na}_{x=0.2} \mathrm{NiPc}, \mathrm{Na}_{x=1} \mathrm{NiPc}$ & $\mathrm{Na}_{x=3} \mathrm{NiPc}$ \\
\hline & $1188 \rightarrow 1193(\mathrm{C}-\mathrm{C}, \mathrm{H}-\mathrm{C}-\mathrm{C})$ & $1452 \rightarrow 1457\left(\mathrm{C}_{\alpha}-\mathrm{N}_{\beta}, \mathrm{C}_{\alpha}-\mathrm{C}, \mathrm{N}_{\alpha}-\mathrm{C}_{\alpha}-\mathrm{N}_{\beta}, \mathrm{C}_{\alpha}-\mathrm{N}_{\beta}-\mathrm{C}_{\alpha}, \mathrm{N}_{\beta}-\mathrm{C}_{\alpha}-\mathrm{C}\right)$ \\
\hline & \multicolumn{2}{|l|}{$1105 \rightarrow 1108\left(\mathrm{C}_{\alpha}-\mathrm{C}, \mathrm{C}-\mathrm{C}\right)$} \\
\hline & \multicolumn{2}{|l|}{$1305 \rightarrow 1309(\mathrm{C}-\mathrm{C}, \mathrm{C}-\mathrm{C}-\mathrm{C})$} \\
\hline & \multicolumn{2}{|c|}{$1467 \rightarrow 1471\left(\mathrm{C}_{\alpha}-\mathrm{N}_{\beta}, \mathrm{C}_{\alpha}-\mathrm{C}, \mathrm{N}_{\alpha}-\mathrm{C}_{\alpha}-\mathrm{N}_{\beta}, \mathrm{C}_{\alpha}-\mathrm{N}_{\beta}-\mathrm{C}_{\alpha}, \mathrm{N}_{\beta}-\mathrm{C}_{\alpha}-\mathrm{C}\right)$} \\
\hline & \multicolumn{2}{|c|}{$1544 \rightarrow 1552\left(\mathrm{C}_{\alpha}-\mathrm{N}_{\beta}, \mathrm{C}_{\alpha}-\mathrm{N}_{\alpha}, \mathrm{N}_{\alpha}-\mathrm{C}_{\alpha}-\mathrm{N}_{\beta}, \mathrm{C}_{\alpha}-\mathrm{N}_{\beta}-\mathrm{C}_{\alpha}, \mathrm{N}_{\beta}-\mathrm{C}_{\alpha}-\mathrm{C}\right)$} \\
\hline & \multicolumn{2}{|c|}{$1607 \rightarrow 1612\left(\mathrm{C}_{\alpha}-\mathrm{N}_{\beta}, \mathrm{C}_{\alpha}-\mathrm{N}_{\alpha}, \mathrm{N}_{\alpha}-\mathrm{C}_{\alpha}-\mathrm{N}_{\beta}, \mathrm{C}_{\alpha}-\mathrm{N}_{\beta}-\mathrm{C}_{\alpha}, \mathrm{N}_{\beta}-\mathrm{C}_{\alpha}-\mathrm{C}\right)$} \\
\hline Новые линии, $\mathrm{cm}^{-1}$ & 636 & $636,723,738,779,1372$ \\
\hline $\begin{array}{c}\text { Иные } \\
\text { отличия }\end{array}$ & \multicolumn{2}{|c|}{ Линия на $1338 \mathrm{~cm}^{-1}(\mathrm{NiPc})$ в спектрах допированных образцов разделяется на две: 1340 и $1346 \mathrm{~cm}^{-1}$} \\
\hline
\end{tabular}

в спектрах допированных образов наблюдались лишь незначительные отличия по сравнению со спектром номинально чистого NiPc. В области $1445-1525 \mathrm{~cm}^{-1}$ для всех допированных образцов наблюдалось некоторое изменение профиля семейства линий. Сравнительный анализ спектров, полученных при $\lambda=633 \mathrm{~nm}$ (рис. 5, $b$ ), приведен в табл. 4. Интерпретация частотных сдвигов, наблюдаемых в полученных спектрах КРС, выполнена на основе результатов работы [20], в которой были проведены расчеты нормальных колебаний молекул металлфталоцианинов и были соотнесены все экспериментальные линии в спектрах КРС.

Перестройка интенсивностей линий и сдвиги некоторых частот позволяют зарегистрировать присутствие в образцах избыточной электронной плотности, которую можно связать с наличием электронов, присоединенных молекулами фталоцианина никеля. Тогда, согласно данным табл. 4, электроны, остающиеся в образцах после допирования их натрием, локализо- ваны, главным образом, вблизи пятичленных циклов $-\mathrm{N}_{\alpha}-\mathrm{C}_{\alpha}-\mathrm{C}_{\beta}-\mathrm{C}_{\beta}-\mathrm{C}_{\alpha}-$ (рис. 4) и шестичленных (ароматических) циклов, состоящих только из атомов углерода. Присутствие в образцах дополнительных электронов также влияет на изменение угла связи $\mathrm{N}_{\alpha}-\mathrm{C}_{\alpha}-\mathrm{N}_{\beta}$, что проявляется в частотных сдвигах соответствующих линий. Возникновение новых линий в спектре КРС может быть объяснено либо изменением условий резонанса (главным образом, для длины волны возбуждающего излучения $\lambda=633 \mathrm{~nm}$ ), вызванным перестройкой электронной структуры молекулы, либо появлением продуктов распада молекул фталоцианина. Поскольку наиболее заметные изменения (четыре новые линии и увеличение/уменьшение интенсивности остальных линий) в спектре КРС проявляются только в образце с наибольшей концентрацией натрия, при которой образуется существенное количество наночастиц никеля, то вторая интерпретация представляется более вероятной. 


\section{Заключение}

Разработана методика допирования парами натрия поликристаллических порошков $\mathrm{NiPc}$ при относительно низких температурах $\left(\sim 300^{\circ} \mathrm{C}\right)$. Просвечивающая электронная микроскопия показала, что в образце $\mathrm{Na}_{x=3} \mathrm{NiPc}$ присутствуют металлические наночастицы со средним диаметром $\sim 20 \mathrm{~nm}$. Сравнение рентгенограмм чистого $\mathrm{NiPc}$ и допированных $\mathrm{Na}_{x=0.2} \mathrm{NiPc}, \mathrm{Na}_{x=1} \mathrm{NiPc}_{2} \mathrm{Na}_{x=3} \mathrm{NiPc}$ фталоцианинов показало наличие наночастиц $\mathrm{Ni}$ лишь в образце $\mathrm{Na}_{x=3} \mathrm{NiPc}$. Наблюдается разупорядочение во взаимной ориентации различных молекул $\mathrm{NiPc} п р и$ стабильной структуре каждой молекулы. В образцах $\mathrm{Na}_{x=0.2} \mathrm{NiPc}, \mathrm{Na}_{x=1} \mathrm{NiPc}$ наночастиц никеля не обнаружено, но установлено наличие локальных структурных искажений в исходной фталоцианиновой матрице. Результаты анализа дифрактограмм подтверждены количественным структурным анализом образцов $\mathrm{Na}_{x} \mathrm{NiPc}$, выполненным с помощью Ni $K$-EXAFS-спектров. Обработка EXAFS позволила определить структурные параметры $\mathrm{Ni}-\mathrm{N}-, \mathrm{Ni}-\mathrm{C}-, \mathrm{Ni}-\mathrm{Ni}$-связей и установить, что в образцах $\mathrm{Na}_{x} \mathrm{NiPc}$ с низкой степенью допирования $(x=0.2,1)$ имеют место локальные искажения исходной фталоцианиновой матрицы, а при увеличении степени допирования до $x=3$ в образце формируются наночастицы Ni, встроенные в матрицу NiPc. Анализ спектров КРС показал, что для слабо допированных образцов $(x=0.2,1)$ имеется дополнительная электронная плотность на пятичленных азотно-углеродных и шестичленных ароматических кольцах фталоцианина, что проявляется в частотном сдвиге соответствующих линий. В случае сильного допирования $(x=3)$ в спектре КРС при $\lambda=633 \mathrm{~nm}$ появляются новые линии, которые можно интерпретировать как колебания атомов $\mathrm{C}$ и $\mathrm{N}$ - продуктов распада фталоцианиновых молекул. Для образца $\mathrm{Na}_{x=3} \mathrm{NiPc}$ полученное из EXAFS значение параметра $C \sim 60 \%$ (доля атомов $\mathrm{Ni}$ в никелевых наночастицах от общего числа атомов $\mathrm{Ni}$ в образце) позволяет заключить, что такое значение обеспечивает наличие устойчивого на воздухе комнатно-температурного ферромагнетизма, сохраняющегося длительное время.

Работа выполнена при поддержке совместного проекта Российского фонда фундаментальных исследований (РФФИ № 15-52-05051 Арм_а) и Государственного комитета по науке Министерства образования и науки Республики Армения (SCS 15 RF-085 51).

Часть российских авторов выражают благодарность проекту Южного федерального университета (ВнГр-07/2017-06).

\section{Список литературы}

[1] Aiken III J.D., Finke R.G. // J. of Mol. Cat. A: Chem. 1999. Vol. 145. P. 1-44.

[2] Reisfeld R., Saraidarov T., Levchenko V. // Opt. Appl. 2008. Vol. 38. N 1. P. 83-92.
[3] Jia C.-J., Schuth F. // Phys. Chem. Chem. Phys. 2011. Vol. 13. N 7. P. $2457-2487$.

[4] Liu C.-X., Liu Q., Guo C.-C., Tan Z. // J. Porphyrins Phthalocyanines. 2010. Vol. 14. P. 825-831.

[5] Idowu M., Nyokong T. // Int. J. Nanosci. 2012. Vol. 11. N 2. P. 1250018.

[6] Guan J.G., Wang W., Gong R.Z., Yuan R.Z., Gan L.H., Tam K.C. // Langmuir. 2002. Vol. 18. N 11. P. 4198-4204.

[7] Brauer B., Vaynzof Y., Zhao W., Kahn A., Li W., ZahnD.R.T., Fernandez C. de Julian, Sangregorio C., Salvan G. // J. Phys. Chem. 2009. Vol. 113. P. 4565-4570.

[8] Schaak R.E., Sra A.K., Leonard B.M, Cable R.E., Bauer J.C., Han Y.-F, Means J., Teizer W., Vasquez Y., Funck E.S. // J. Am. Chem. Soc. 2005. Vol. 127. N 10. P. 3506-3515.

[9] Li F., Yu X., Pan H., Wang M., Xin X. // Sol. Stat. Sci. 2000. Vol. 2. N 8. P. 767-772.

[10] Колпачева Н.А., Авакян Л.А., Манукян А.С., Мирзаханян А.А., Шароян Е.Г., Прядченко В.В., Зубавичус Я.В., Тригуб А.Л., Федоренко А.Г., Бугаев Л.А. // ФТТ. 2016. Т. 58. Вып. 5. С. 972-978.

[11] Zhou S., Li Y., Chen Z., Li X.X., Chen N., Du G. // Ceramics International. 2013. Vol. 39. N 6. P. 6763-6768.

[12] Grigoryan L., Simonyan M., Sharoyan E. Patent of USSR N 120686. 1984.

[13] Robertson J.M., Woodward I. // J. Chem. Soc. 1937. Vol. 36. P. 219.

[14] Chichagov A.V., Varlamov D.A., Dilanyan R.A., Dokina T.N., Drozhzhina N.A., Samokhvalova O.L., Ushakovskaya T.V. // Cryst. Rep. 2001. Vol. 46. N 5. P. 876-879.

[15] Pryadchenko V.V., Srabionyan V.V., Mikheykina E.B., Avakyan L.A., Murzin V.Y., Zubavichus Y.V., Zizak I., Guterman V.E., Bugaev L.A. // J. Phys. Chem. C. 2015. Vol. 119. P. 3217-3227.

[16] Srabionyan V.V., Bugaev A.L., Pryadchenko V.V., Makhiboroda A.V, Rusakova E.B., Avakyan L.A., Schneider R., Dubiel M., Bugaev L.A. // J. Non-Crys. Sol. 2013. Vol. 382. P. 24-31.

[17] Newville M., Ravel B., Haskel D., Rehr J.J., Stern E.A., Yacoby Y. // Physica B: Condensed Matter. 1995. Vol. 208209. P. $154-156$.

[18] Koningsberger D.C., Mojet B.L., vanDorssen G.E., Ramaker D.E. // Topics Catalys. 2000. Vol. 10. N 3. P. 143155.

[19] Poiarkova A.V., Rehr J.J. // Phys. Rev. B. 1999. Vol. 59. P. 948.

[20] Melendres C.A., Maroni V.A. // J. Raman Spectroscopy. 1984 Vol. 15. N 5. P. 319-326.

[21] Basova T.V., Kolesov B.A., Gürek A.G., Ahsen V. // Thin Solid Films. 2001. Vol. 385. P. 246-251.

[22] Александров И.В., Бобович Я.С., Маслов В.Г., Сидоров А.Н. // Опт. и спектр. 1974. Т. 37. Вып. 3. С. 467-475. 